\title{
Anisotropic optical and conductive properties of oriented 1D-nanoparticle thin films made by spray-assisted self-assembly
}

\author{
S. Sekar, ${ }^{a, b}$ V. Lemaire, ${ }^{a, b}$ H. Hu, ${ }^{a, b}$ G. Decher ${ }^{a, b, c, d^{*}}$ and M. Pauly ${ }^{a, b^{*}}$ \\ We report on the fabrication of oriented anisotropic metal nanoparticle thin films made by Grazing Incidence Spraying (GIS) \\ and on the anisotropic plasmonic properties of the resulting thin films. Gold nanorods of two different aspect ratios and \\ silver nanowires were self-assembled as a uniaxially aligned monolayer with the GIS approach. In particular, we examine the \\ influence of the nanowire/nanorod length and diameter on the degree of ordering determined on electron microscopy \\ pictures. Furthermore, we show that the anisotropy of the optical properties (probed by polarized UV-Visible-Near InfraRed \\ spectroscopy) strongly depend on the quality of alignment. The prepared monolayer thin films have an orientation order \\ parameter of up to 0.83 for silver nanowires, which reflects in an optical anisotropy of 0.57 in the UV-Visible and 0.76 in the \\ near infrared through the selective excitation of transverse and longitudinal surface plasmon resonance modes. The \\ electronic transport in oriented silver nanowire monolayers is also shown to be highly directional, with the sheet resistance \\ varying over almost an order of magnitude depnding on the transport direction. Such anisotropic conductive plasmonic thin \\ films may find applications in various fields like biochemical sensing, energy transport and harvesting or optoelectronic \\ devices.
}

Corresponding authors email : matthias.pauly@ics-cnrs.unistra.fr ; decher@unistra.fr

\section{Introduction}

Anisotropic materials possess physical properties, for example optical, electronic or mechanical properties that differ in different directions of space. ${ }^{1,2}$ While materials science has made enormous progress over the last decades, especially with respect to composites and nowadays also nano-composites, the methods available for preparing materials that are anisotropic at the nano- to micro-scale are still quite limited. Another roadblock in materials science lies in the controlled preparation of self-assembled materials composed of more than two components, and this holds especially true for nanoscale multicomposites. This is due to the fact that phase separation phenomena at the micro- and nanoscale often prevent to organize the individual components in a composite with precise distances between them. One strategy for building wellcontrolled multimaterial thin films is the so-called Layer-byLayer (LbL) assembly approach,3, 4 in which the sequence of different materials and the distances between them are precisely controlled along the layer normal. ${ }^{5-7}$

Particularly interesting components for the preparation of anisotropic multicomponent materials are one-dimensional (1D-) nanoparticles, such as nanofibrils, nanowires or nanorods whose physical properties can easily be tuned by varying their aspect ratio. ${ }^{1,2}$ While the physical properties of such nanoobjects are often highly anisotropic at the single particle level, their controlled integration into multicomposites is even more difficult than the integration of their spherical counterparts. This is due to the fact that one does not only have to control the dispersion of the particles in a for example polymeric matrix, but to do so while additionally controlling the anisotropy of the composite.

In the present manuscript we use metallic nanorods and nanowires as anisotropic particles, because the anisotropy of nanocomposites of metallic components can easily be probed optically and electrically. Especially, the geometry dependent optical response of the electron cloud of metallic nanorods and -wires, which gives rise to distinct so-called surface plasmon resonances (SPR) along and perpendicular to their long axis, and the fact that the local plasmons of different rods or wires can couple as a function of their distance makes them valuable probes for studying anisotropic materials properties. In particular, metallic nanowires and nanorods have at least two absorbance bands which can be associated to the resonant oscillation within the nanoparticle of metallic electrons along the long axis (longitudinal SPR mode, LSPR) or along the short axis (transverse SPR mode, TSPR). ${ }^{8}$

Complementarily, the direction dependent electrical conductivities of composites depend on macroscopic percolation pathways arising from a network of nanowire/nanowire or nanorod/nanorod contacts across the entire film. Consequently, both the plasmonic properties and the electrical conductivities depend on the in-plane densities and on the degree of alignment of the nanorods and -wires. However, plasmonic and polarized plasmonic phenomena arise predominantly from coupling occurring over some tens of nanometers whereas electrical conduction depends on the homogeneity of a composite at the macroscopic scale. Depending on the percolation geometry, a single break in the millimeter to centimeter long chain of wire/wire contacts between the test electrodes can be sufficient to interrupt percolation completely and will thus greatly reduce electrical conductivities. In this perspective one needs less wire/wire contacts in the direction parallel to the nanowire alignment in order to establish a percolation pathway than in the direction perpendicular to the alignment direction, which can be probed by measuring the directional conductivity of the thin films.

There are numerous applications in which anisotropic properties are needed, ${ }^{9}$ and this interesting anisotropy in the properties at macroscopic scales requires the nanoparticles to be deposited as an oriented thin film over macroscopic distances. One strategy in order to tackle this issue is to set up self-assembly approaches that lead to large arrays of highly 
ordered structures. Indeed, bottom-up fabrication offers great advantages as compared to top-down approaches for the new generation of functional devices that will include nanoparticle building blocks.9-11 Oriented self-assembly is especially important for plasmonic particles, as it allows fine-tuning the so-called hotspots, ${ }^{12-15}$ which are regions (often located at the gap between nanoparticles) in which the high electromagnetic field created by coupled SPR modes in neighbouring particles enhances intrinsic properties of an analyte, such as the Raman absorption bands in Surface Enhanced Raman Scattering (SERS). ${ }^{16}$. Many other applications also make use of this localized amplified field, for example in optoelectronics, photonics and nanoplasmonics as waveguides, optical filters and sensors. ${ }^{17,} 18$ These exciting properties indeed attracted a huge research effort these last years on the (bottom-up) fabrication of plasmonic nanoparticle arrays. ${ }^{2,19}$

A variety of methods have been proposed in order to build selfassembled oriented arrays of nanomaterials, including Langmuir-Blodgett, ${ }^{20}$ electric $^{21}$ or magnetic ${ }^{22}$ fields, microfluidic-assisted deposition, ${ }^{23}$ mechanical stretching, ${ }^{24}$ blown-bubble-assisted ordering ${ }^{25,} 26$ and spray coating ${ }^{27}$. While these techniques have proven to be efficient for the orientation of specific types of nanowires, they all suffer from several drawbacks, such as complex substrate preparation, multistep processes, use of external fields, deposition on small areas only, etc... For keeping the alignment method as general as possible we have recently developed grazing incidence spraying as a LbLcompatible technique based on liquid shear for aligning anisotropic nanoparticles with moderate to high aspect ratios in the form of monolayers on surfaces, ${ }^{28,29}$ a first example using cellulose nanofibrils ${ }^{30}$ was recently reported in more detail. This unique approach overcomes the drawbacks of existing methods and offers the greatest advantages to prepare oriented monoand multilayer thin films in combination with the wellestablished Layer-by-Layer technique. ${ }^{3}, 31$ Additionally, the deposition density and thereby the plasmonic coupling in mono and multilayer can be independently well controlled through easily adjustable spray parameters.

In this article, we present the construction of oriented monolayer thin films of anisotropic metallic nanoparticles of increasing length (gold nanorods (AuNRs) of two different aspect ratios and silver nanowires (AgNWs)) using the GIS approach. As the orientation is due to shear forces exerted by the flowing liquid on the nano-objects, we show that the degree of orientation increases with the length of the anisotropic material. The resulting thin films show strong anisotropic optical and electronic transport properties, and the optical anisotropy reflects the degree of ordering of the thin films.

\section{Results and Discussion}

\section{Characterization of anisotropic nanoparticles}

1D nanomaterials were synthesized following protocols previously reported in the literature ${ }^{32-34}$ with slight modifications (for details, see experimental section). The TEM and SEM images clearly demonstrate the successful synthesis of AuNRs and AgNWs (Fig. 1A). The length and diameter distributions are given in Fig. $1 \mathrm{~B}$ and Table 1. The relative size distribution spans from $10-25 \%$ for the various nanoparticles. Despite of this length distribution, the three different nanoobjects used here have very distinguishable length, ranging from $\sim 130 \mathrm{~nm}$ for the Short Au NanoRods (SAuNRs), $250 \mathrm{~nm}$ for the Long Au NanoRods (LAuNRs) and up to $\sim 5.7 \mu \mathrm{m}$ for the AgNWs.

Only very few spherical impurities are seen in the SAuNRs, whereas other shapes such as nanoplates, triangles and short nanorods are present in the LAuNRs suspension besides long nanorods. The fraction of shape impurities other than nanorods has been estimated to $\sim 10 \%$ for SAuNRs and $~ 15 \%$ for LAuNRs, while only nanowire are present in AgNWs. The increasing size and shape distribution for high aspect ratio gold nanorods is a well-known generic problem faced in the synthesis of high aspect ratio rods. ${ }^{33,} 35 \mathrm{~A}$ closer look to both short and long nanorods TEM images reveal that the rods self-assemble during the TEM-grid preparation as a very dense array triggered by a strong side-by-side packing. ${ }^{36}$ 
A

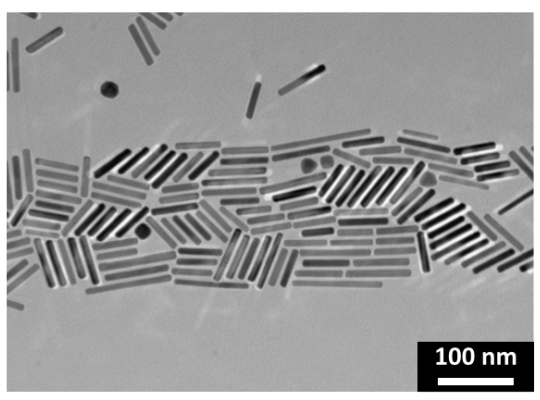

B
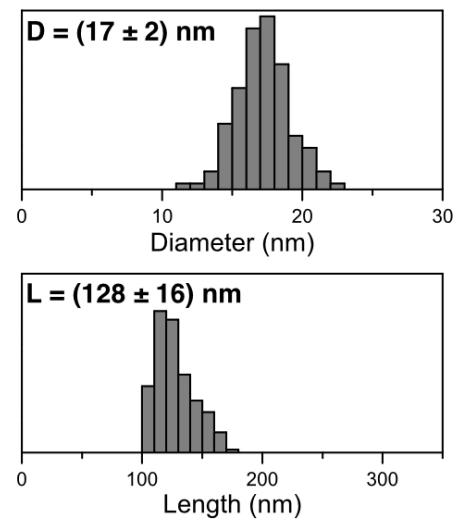

$\mathbf{C}_{3}$

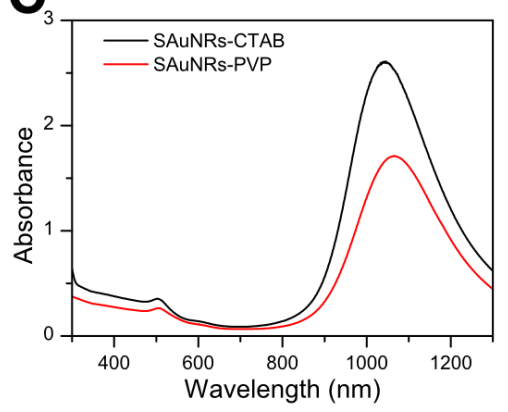

LAuNRs
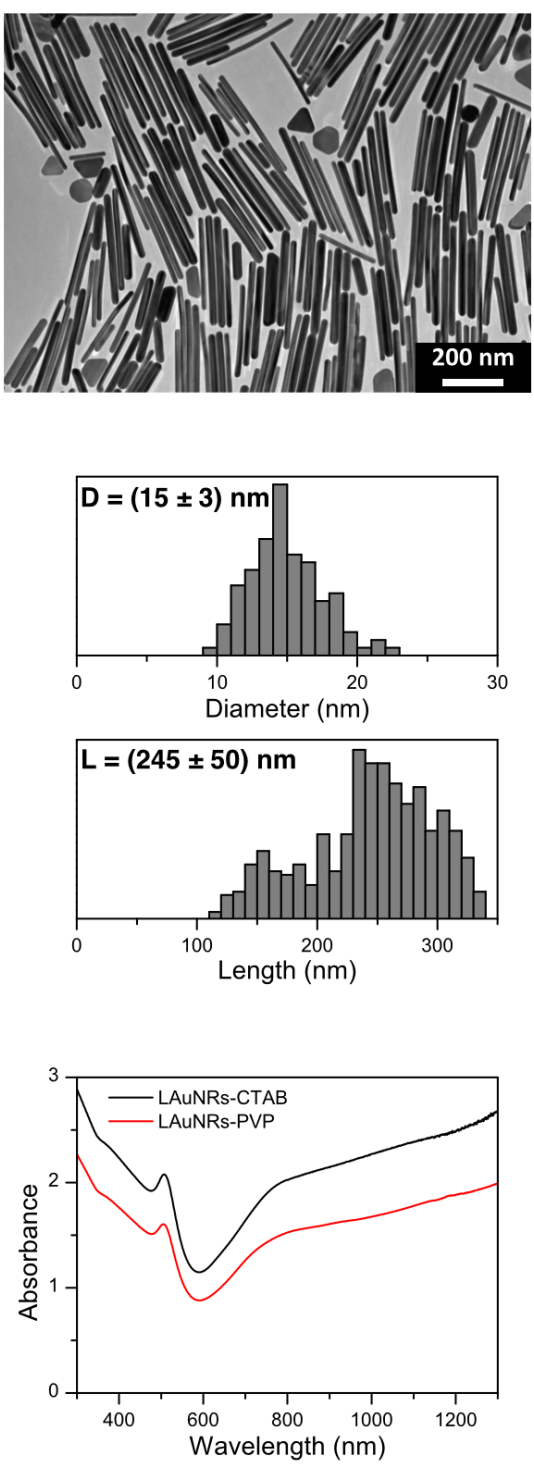

AgNWs
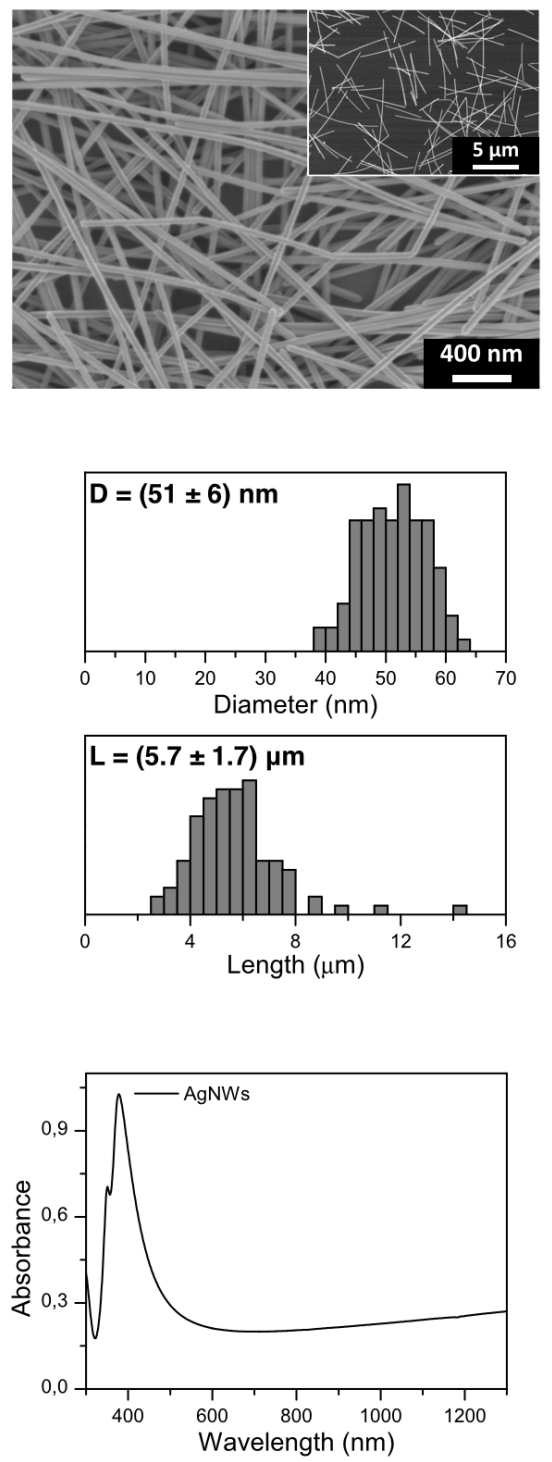

Figure 1: Electron Microscopy (A), diameter and length distribution (B), and UV-Vis-NIR spectra (C) of the nanoparticle suspensions for SAuNRs (AR 7, left panels), LAuNRs (AR $\sim 16$, middle panels) and AgNWs (right panels). The images have been acquired by TEM for AuNRs and by SEM for AgNWs. The size distributions are measured on $>100$ nanoparticles. The spectra before and after ligand exchange with PVP are measured in water for the AuNRs suspensions and in ethanol for the AgNWs.

The optical spectra of both AuNRs and AgNWs are presented in Fig. 1C. While small spherical metal nanoparticles usually exhibit only one single SPR absorption band, more modes can be excited in anisotropic nano-objects. For instance, at least two resonance modes can be excited in 1D nanoparticle such as nanowires and nanorods: 8 the first one, associated to the Transverse dipolar oscillations along the short axis of the nanorod (TSPR), appears at $503 \mathrm{~nm}$ for the SAuNRs and at 507 $\mathrm{nm}$ for the LAuNRs. Two TSPR bands are observable for the AgNWs at $349 \mathrm{~nm}$ and $378 \mathrm{~nm}$, associated to two different transverse modes for $\mathrm{Ag}$ nanowires with a pentagonal cross section. ${ }^{37}$ Indeed, nanowires with non-circular cross-sections are known to display multiple resonance modes, and the two bands correspond to the out-of-plane quadrupole resonance and dipole resonance of nanowires respectively. ${ }^{38-40}$ The resonance band associated to the Longitudinal Surface Plasmon
Resonance (LSPR) mode along the long axis of the nanorod/nanowire appears in the near Infrared (NIR). The position of this LSPR band is known to be highly dependent on the shape and size of the nanocrystals, and the absorption band red-shifts and broadens with increasing aspect ratio. ${ }^{41,} 42$ Indeed, the LSPR of the SAuNRs reflects as a broad absorbance peak centered at $1040 \mathrm{~nm}$. For the higher aspect ratio LAuNRS

Table 1: length, diameter and aspect ratio of AuNRs and AgNWs.

\begin{tabular}{|c|c|c|c|}
\hline & Length $(\mathbf{n m})$ & Diameter $(\mathbf{n m})$ & Aspect Ratio \\
\hline SAuNRs & $128 \pm 16$ & $17 \pm 2$ & $\sim 7$ \\
\hline LAuNRs & $245 \pm 50$ & $15 \pm 3$ & $\sim 16$ \\
\hline AgNWs & $(5.7 \pm 1.7) \times 10^{3}$ & $51 \pm 6$ & $\sim 110$ \\
\hline
\end{tabular}


and for AgNWs, the longitudinal band(s) appears in the NIR range, at a wavelength that is beyond the investigated spectral range. Indeed, the solvent absorption around $1300 \mathrm{~nm}$ does not allow for a clear measurement of the nanoparticle absorbance band.

As spherical nanoparticle impurities have their absorbance band in the same range as the TSPR of the nanorods, the high absorbance intensity ratio of 7.6 of LSPR to TSPR of SAUNRs indicates that the contribution of the spherical impurities on the optical absorbance spectrum is low. ${ }^{32,43}$ Furthermore, the LSPR peak position depends on the diameter and length, and it has been shown empirically 32,44 that the position of the LSPR band of gold nanorods $\lambda_{\text {LSPR }}$ varies linearly with the aspect ratio (AR):

$$
\begin{array}{ll}
\lambda_{L S P R}=95 A R+420 \quad \text { Eq. } 1
\end{array}
$$

This leads to an AR estimated from the optical properties of 6.5, which is in good agreement with the AR determined from TEM measurements ( 7 ). The same correlation can not be made for the higher AR nano-objects as the $\lambda_{\text {LSPR }}$ can not be measured. For the LAUNRs, in addition to the TSPR at $507 \mathrm{~nm}$ and the broad LSPR extending towards the NIR, one can notice a broad shoulder around $800 \mathrm{~nm}$ that can be related to the presence of shape-impurities, such as platelets and triangles. ${ }^{33}$ Several strategies have been proposed in order to selectively remove those shape impurities. ${ }^{45-47}$ However, the shape selectivity is low and we had to apply several purification cycles in order to increase significantly the nanorod to impurity ratio, which drastically reduced the final synthesis yield. For this reason, we choose to use the LAuNRs as synthesized and without any size/shape selection.

AgNWs are coated in-situ by polyvinylpyrrolidone (PVP), ${ }^{48}$ whereas AuNRs are stabilized by a Cetyl TrimethylAmmonium Bromide (CTAB) bilayer. ${ }^{49}$ Exchange of the CTAB bilayer coating by PVP is requisite to facilitate the adsorption of AuNRs. Indeed, Quartz Crystal Microbalance (QCM) experiments (see SI, Fig. S1) show that the as-prepared positively charged CTAB capped AuNRs failed to adsorb on the oppositely charged quartz substrates. This might be due to the preferential adsorption of the free CTAB present in the suspension on the substrate which hinders the further deposition of AuNRs and thus leads to lower AuNRs coverage. As there is a fast equilibrium between free CTAB in AuNRs dispersions and CTAB adsorbed on the NRs surfaces, a complete removal of free CTAB would require repeated centrifugation cycles, which induces the destabilization of the colloidal dispersion. Therefore, we choose to rather coat the AuNRs with PVP that provides the stability through steric interactions. PVP functionalization provides sufficient stability to the AuNRs to maintain their colloidal stability without any aggregation for several months. TEM measurements (SI, Fig. S2) shows a $2.5 \mathrm{~nm}$ thick PVP coating on the rod surface. The optical spectra of both SAuNRs and LAuNRs after PVP functionalization show a slight red shift in comparison to the native CTAB coating. For instance, the LSPR band of the SAuNRs shifts from 1040 to $1067 \mathrm{~nm}$. This can be ascribed to the local changes in the refractive index due to the PVP coating in the vicinity of the particle surface and to a small extent to the different solvent composition, as $\sim 20$ vol\% ethanol is present in the AuNRs aqueous suspension after surface modification (see Experimental section). ${ }^{50,51} \mathrm{QCM}$ experiments demonstrate that the adsorption of AuNRs on the substrate is successful after they have been coated by PVP.

Following the recent reports by Polte and Liz-Marzan, ${ }^{52,53}$ the $\left[\mathrm{Au}^{0}\right]$ concentration in SAuNRs-PVP and LAuNRs-PVP is deduced from the absorbance intensity at $400 \mathrm{~nm}$ and for all our experiments the concentration of $\left[\mathrm{Au}^{0}\right]$ in water was set to $0.5 \mathrm{mM}$. AgNWs were dispersed in ethanol at a concentration $\left[\mathrm{Ag}^{0}\right]$ of $0.1 \mathrm{mM}$.

\section{Oriented monolayer deposition of anisotropic metal nanoparticles}

As depicted in Fig. 2A, we used Grazing Incidence Spraying (GIS) in order to build monolayer thin films in which the 1Dnanoparticles are oriented along the spraying direction. As we have shown recently, ${ }^{29}, 30$ this shear-induced alignment technique is very simple, versatile and applicable to various types of anisotropic materials. In the present case, PVP coated AuNRs suspensions $\left[\mathrm{Au}^{0}=0.5 \mathrm{mM}\right.$ ] and $\mathrm{AgNW}$ s suspensions $\left[\mathrm{Ag}^{0}=0.1 \mathrm{mM}\right]$ were sprayed on polyethyleneimmine-coated glass substrate at respective air flow rates of $40 \mathrm{~L} / \mathrm{min}$ and $30 \mathrm{~L} / \mathrm{min}$ and at a liquid flow rate of $1 \mathrm{~mL} / \mathrm{min}$ for 250 seconds with a constant angle of $15-20^{\circ}$ and distance of $1 \mathrm{~cm}$ between the receiving substrate and spraying nozzle.

The deposited layers were imaged by SEM (Fig. 2B), and the images were analysed using the OrientationJ plugin ${ }^{54}$ developed for Image ${ }^{55}$ (see experimental section for details). During this process, each pixel is color-coded according to its orientation angle (Fig. 2C). The angular distribution of the nanorods/nanowires orientation can thus be measured (Fig. 2D). In order to compare the quality of alignment between the different samples, we calculated a 2D nematic order parameter $S_{\text {microscopy }}$ from microscopy images, defined as:

$$
S_{\text {microscopy }}=<2 \operatorname{Cos}^{2} \theta-1>>\quad \text { Eq. } 2
$$

where $\theta$ is the angle between the anisotropic nanoparticle orientation direction and the main orientation direction as measured on electron microscopy pictures. Indeed, $S_{\text {microscopy }}=0$ for an isotropic ensemble of 1D-nanoparticles and $S_{\text {microscopy }}=1$ for a perfectly oriented system.

As the spray jet is directed with a low angle towards the substrate, the nano-object suspension is forming a constantly flowing liquid film at the substrate/suspension interface, which induces a shear force on the 1D-nanoparticles and orient the nanorods/nanowires along the spraying direction during the adsorption process. As the shear force exerted by a viscous liquid on an object scales with the interface area, one can expect that the shear force and thus the degree of ordering increases with the length of the deposited object. Indeed, this effect can clearly be observed on Fig. 2: the SAuNRs $(L=130 \mathrm{~nm})$ show a very low orientation $\left(S_{\text {microscopy }}=0.15\right)$, whereas the longer nanorods LAuNRs $(L=250 \mathrm{~nm})$ are much better oriented $\left(S_{\text {microscopy }}=0.61\right.$, i.e. that $48 \%$ of the LAuNRs are oriented within $\pm 15^{\circ}$ from the main direction). The presence of shape impurities may partially impair the alignment and probably limits the ordering quality. Furthermore, these shape impurities are less anisotropic than the nanorods, and this 
results in a broadening of the orientation angle distribution determined from the microscopy pictures. Finally, a further increase of the AgNWs length to the micrometer range $(L=5.7$ $\mu \mathrm{m}$ ) allows reaching a high order parameter of $\mathrm{S}_{\text {microscopy }}=0.83$, which corresponds to $73 \%$ of the AgNWs being oriented at $\pm 15^{\circ}$ from the main direction. It must also be noted that all the nanoparticles used in this study have the same surface functionalization, as all of them are coated with PVP, which excludes differences that could arise from a different surface chemistry of the nanoparticles and thus a different interaction with the PEl-functionalized substrate.

A recent report by Assad et al. ${ }^{27}$ on a spray coating route used to orient Si nanowires shows that the quality of alignment highly depends on the compatibility of droplet size with the dimension of nanomaterials and the temperature of the receiving substrate. However, the process these authors describe is very different from what we present here, as the orientation does not occur upon droplet evaporation as for their study but we
A

\section{B}

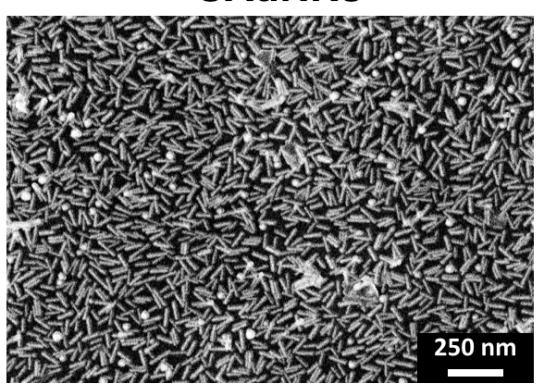

C

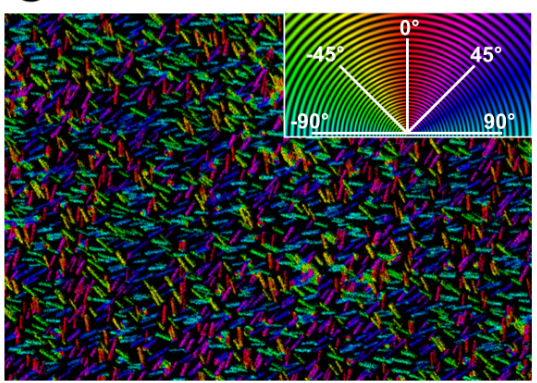

D

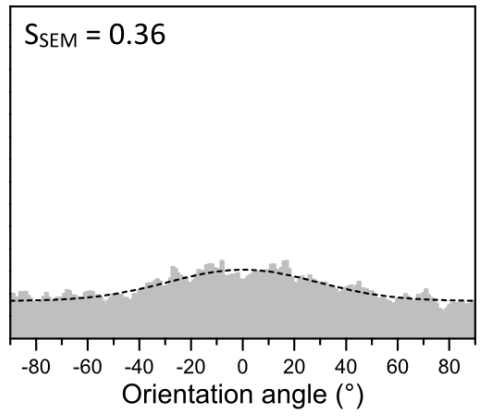

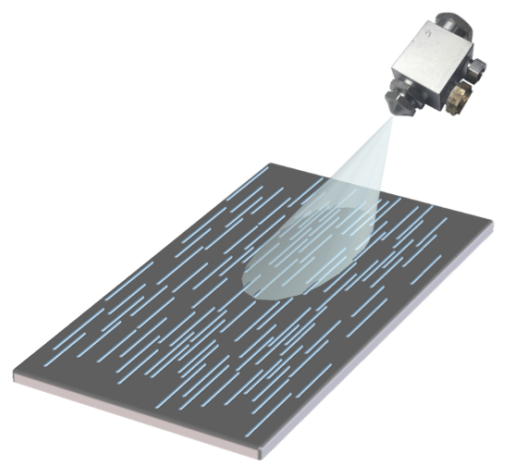

LAuNRs
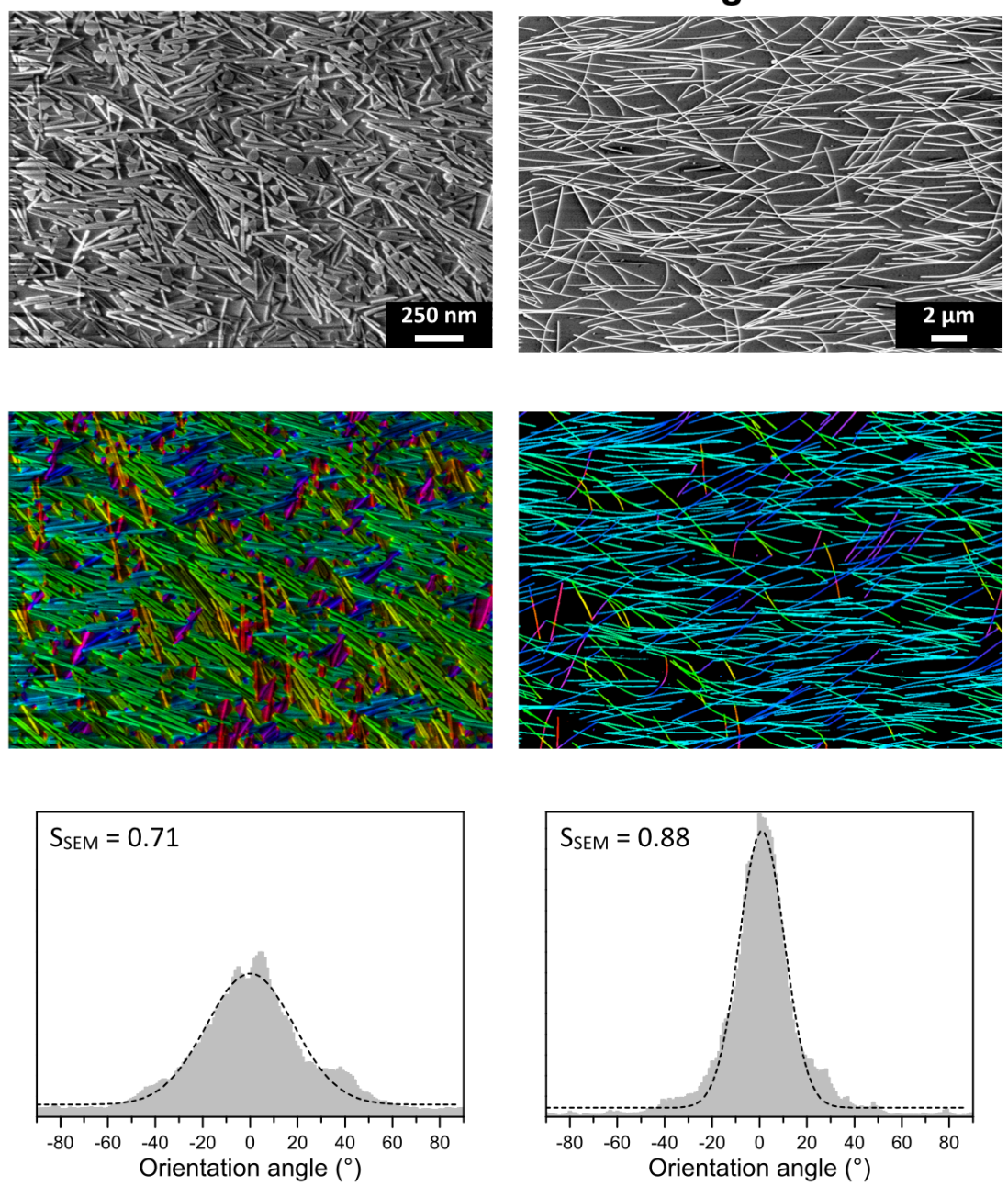

AgNWs
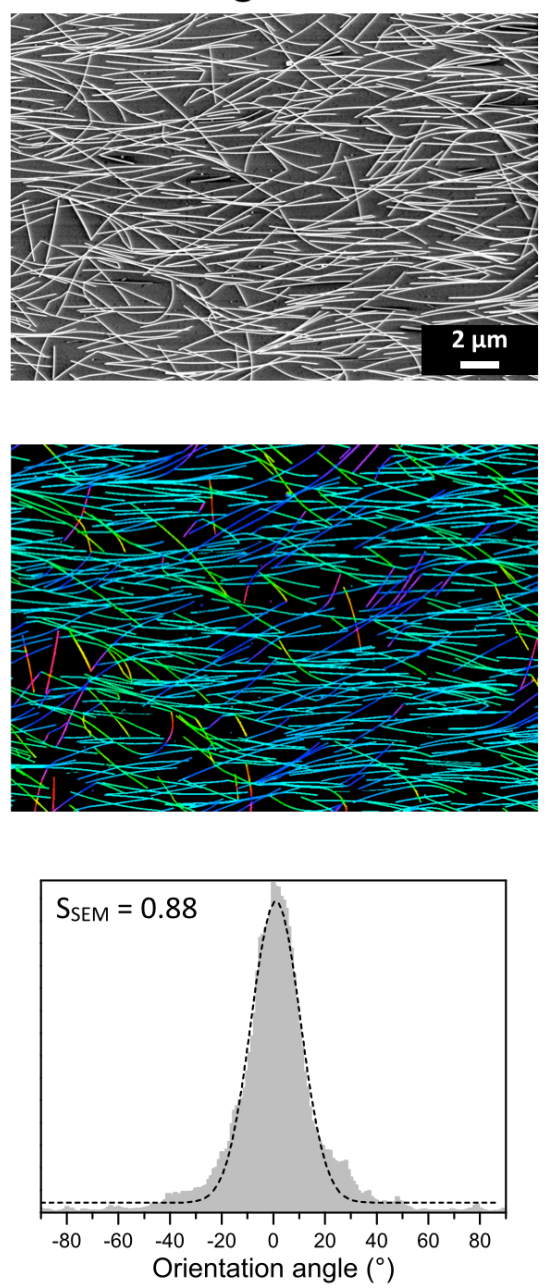

Figure 2: A) Schematic representation of the GIS approach. B) SEM picture of a deposited monolayer, C) orientation analysis in which the nanoparticles are coloured according to their orientation and D) angle distribution with respect to the spraying direction (the dashed line represents a gaussian fit of the distribution, $\mathrm{S}_{\text {microscopy }}$ is the nematic order parameter, see Eq. 2) of SAuNRs (left panel), LAuNRs (middle panel) and AgNWs (right panel) deposited on PEl coated glass substrate. 

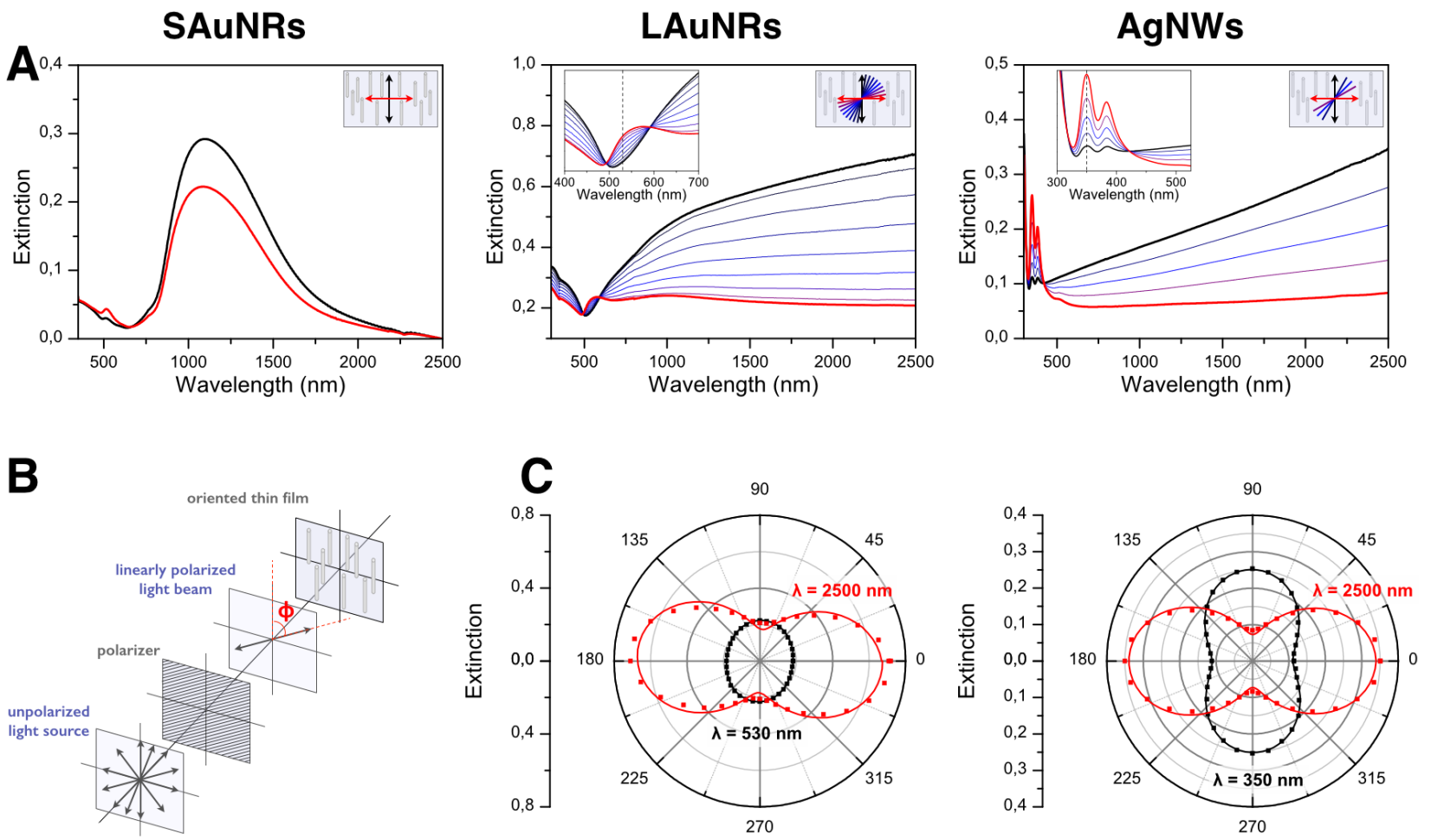

Figure 3: A) UV-Vis-NIR extinction spectra of SAuNRS-PVP (left panel), LAuNRS-PVP (middle panel) and AgNWs (right panel) oriented thin films for a varying polarizing angle $\phi$, B) schematic representation of the polarized UV-Vis-NIR set-up, in which $\phi$ (polarizing angle) is defined as the angle between the orientation direction in the film and the polarization direction of the incident light, and C) polar plot of the extinction of the LAuNRs-PVP (middle) and the AgNWs (right) thin films as function of $\phi$ in the UV-Vis (black curves) and in the NIR (red curve). The line is a fit to a sine square function.

rather keep a liquid film wetting the substrate at all time during the orientation process.

\section{Optical properties of plasmonic thin films}

As shown previously, we have been able to assemble the $1 \mathrm{D}$ nanoparticle as a single monolayer in which the nanowire/nanorods are oriented and point all in a single direction. As we have seen in Fig. 1C, the plasmonic 1Dnanoparticles have at least two plasmon modes located in the UV-Vis and NIR that are linked to the short and long axis of the nanoparticles respectively. The anisotropy in the nanoparticle ordering induced by the GIS approach should thus reflect in an anisotropy in the optical properties. This has been probed by measuring the polarized UV-Vis-NIR extinction spectra, and by varying the polarizing angle $\phi$ between the incident light polarization plane and the nanoparticle orientation direction in the thin film (Fig. 3B). For a perfectly oriented thin film, it can be expected that the LSPR and the TSPR modes can be excited selectively, which means that the extinction spectra should vary drastically with the polarizing angle. 8,24 This is indeed what is observed for all the polarized spectra to different extends (Fig. $3 \mathrm{~A})$ : when light is polarized along the long axis of the nanorods/nanowires $\left(\phi=0^{\circ}\right.$, black curves), the LSPR band is excited and gives rise to a broad extinction in the NIR, whereas the TSPR band is excited in the UV-Vis when light is polarized along their short axis $\left(\phi=90^{\circ}\right.$, red curves). All three samples presented here thus show a certain polarizing property, the direction of polarization being orthogonal in the UV-Vis and in the NIR range as they are linked to two orthogonal SPR modes.
The SAuNRs-PVP spray-coated thin film (Figure 3A) measured at a polarizing angle of $\phi=0^{\circ}$ and $90^{\circ}$ shows only a small difference between the two spectra. This indicates that the anisotropy of the optical properties is low, as expected from the low degree of ordering of the nanoparticles $\left(S_{\text {microscopy }}=0.15\right)$. A slight red shift and a significant broadening of both TSPR and LSPR peaks as compared to the suspension is also observed, which is due to the inter-particle coupling. ${ }^{50}$ For instance, the TSPR band shifts from $1067 \mathrm{~nm}$ for the suspension to $\sim 1080 \mathrm{~nm}$ for the monolayer.

As the degree of ordering increases for the LAuNRs $\left(\mathrm{S}_{\text {microscopy }}=\right.$ $0.61)$ and for the AgNWs $\left(S_{\text {microscopy }}=0.83\right.$ ) thin films, the anisotropy in the optical properties is more and more obvious, as shown by the increasing difference between the polarized spectra at $\phi=0^{\circ}$ and $90^{\circ}$. The polarized spectra for a varying polarizing angle have been measured (Fig. $3 \mathrm{~A}$, middle and right panels), and the extinction at selected wavelength have been represented as a polar plot as function of the polarizing angle $\phi$

Table 2: nematic order parameter $S_{\text {microscopy }}$ and optical order parameter $S_{\text {opt }}$ measured close to the TSPR and to the LSPR for the oriented SAuNRs, LAuNRs and AgNWs oriented thin films

\begin{tabular}{|c|c|c|c|}
\hline & S $_{\text {microscopy }}$ & Sopt $_{\text {(TSPR) }}$ & S opt $_{\text {(LSPR) }}$ \\
\hline SAuNRs & 0.15 & $0.32 @ 527 \mathrm{~nm}$ & $0.30 @ 1700 \mathrm{~nm}$ \\
\hline LAuNRs & 0.61 & $0.18 @ 530 \mathrm{~nm}$ & $0.71 @ 2500 \mathrm{~nm}$ \\
\hline AgNWs & 0.83 & $0.57 @ 350 \mathrm{~nm}$ & $0.76 @ 2500 \mathrm{~nm}$ \\
\hline
\end{tabular}


(Fig 3C). These polar plots are given at the wavelength close to the TSPR at which the polarization is highest $(\lambda=530 \mathrm{~nm}$ for the LAuNRs and $\lambda=350 \mathrm{~nm}$ for the AgNWs, see the dashed line in the insets of Fig. 3A). As the LSPR peak is beyond the investigated spectral range, we have plotted the NIR-extinction at $2500 \mathrm{~nm}$ for both LAuNRs and AgNWs. The polar plots evidence clearly that the extinction in the UV-Vis is maximum at $\phi=90^{\circ}$ when the transverse modes are excited, whereas it reaches its minimum at $\phi=0^{\circ}$. On the contrary, the extinction in the NIR is maximum at $\phi=0^{\circ}$ (i.e. that the LSPR is selectively excited) and minimum at $\phi=90^{\circ}$. The variation of the extinction fits well with a sine square function.

For the LAuNRs thin film in the UV-Vis (inset of Fig. 3C), the apparent position of the TSPR peak is red-shifted as compared to the suspension $(\lambda=507 \mathrm{~nm})$ and varies with the polarizing angle. For instance, the "peak" appears at $\sim 570 \mathrm{~nm}$ for $\phi=90^{\circ}$; This can be explained by a plasmonic coupling along the short axis of neighbouring rods when light is polarized perpendicularly to the alignment direction.

It can also be further observed that the spectra are very sensitive to the polarization direction in NIR range for both the LAuNRs and the AgNWs, whereas the behaviour is different in the UV-Vis range: the TSPR bands are very sensitive to the polarizing angle for the AgNWs but it is much less responsive for the LAuNRS. This can be clearly observed on the polar plots: the extinction as function of $\phi$ in the UV-Vis and in the NIR ranges are of the same order of magnitude for the AgNWs, whereas the extinction for the LAuNRs samples in the UV-Vis is much lower and less responsive to the variation of $\phi$ than the extinction in the NIR. This weak amplitude and low polarization is caused by the broadening of the TSPR peak due to its overlap with interband transition of metallic gold at $\sim 400 \mathrm{~nm} .{ }^{8}$

The optical anisotropy can be quantified using an optical order parameter $\mathrm{S}_{\text {opt }}$ defined as:

$$
S_{\text {opt }}=1-\frac{A_{\min }}{A_{\max }} \quad \text { Eq. } 3
$$

with $A_{\min }=A_{90^{\circ}}$ and $A_{\max }=A_{0^{\circ}}$ for the LSPR and $A_{\min }=A_{0^{\circ}}$ and $A_{\max }=A_{90}$, for the TSPR. $S_{\text {opt }}$ is thus a direct measurement of the polarisation efficiency as $\mathrm{S}_{\mathrm{opt}} \longrightarrow 0$ for an isotropic sample (i.e. when $\left.A_{\min } \sim A_{\max }\right)$, whereas $S_{\text {opt }} \longrightarrow 1$ for a highly anisotropic sample (i.e. when $A_{\min } \ll A_{\max }$ ). The values of the optical order parameter $S_{\text {opt }}$ are summarized in Table 2 and compared to the 2D nematic order parameter determined from the SEM pictures $\mathrm{S}_{\text {microscopy }}$. The optical anisotropy increases with the nematic order parameter, reaching values higher than 0.7 for the LAuNRs and the AgNws thin films in the NIR. This confirms that the optical anisotropy is directly linked to the order parameter. It must be noted that as the peak of the LSPR is beyond the investigated wavelength range, the optical anisotropy further increases for $\lambda>2500 \mathrm{~nm}$, and the maximum of optical anisotropy is thus probably much greater than 0.7 further in the NIR. However, the optical anisotropy close to the transverse band for the LAuNRs $\left(\mathrm{S}_{\mathrm{opt}}=0.18\right.$ ) is lower than for the SAuNRs and for the AgNWs. This means that the extinction is less responsive to the variation of $\phi$, because the overlap of the TSPR with the gold interband transitions that are not dependent on the polarization state of the incoming light.

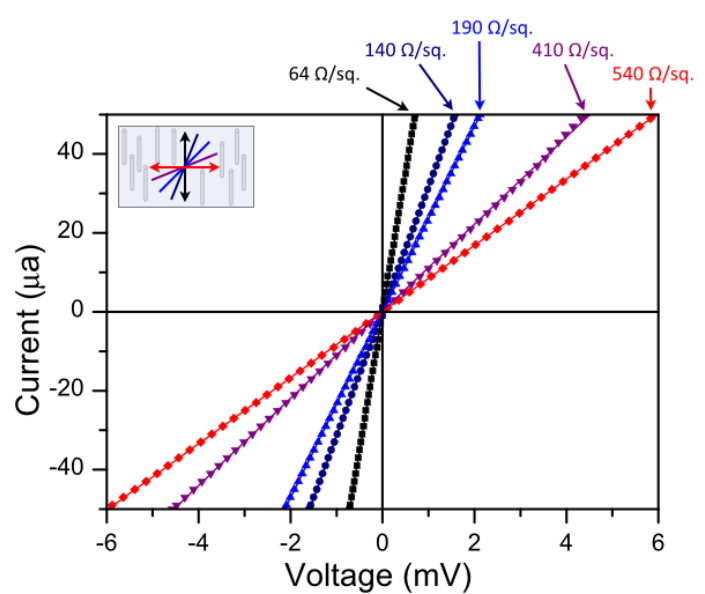

Figure 4: current as function of the applied voltage measured in a 4-probe configuration on a AgNW monolayer in different directions with respect to the orientation direction. The sheet resistance is $>8$ times lower along the nanowire orientation direction compared to the orthogonal direction.

Finally, it must be noted that although the same concentration of $\left[\mathrm{Au}^{0}\right]=0.5 \mathrm{mM}$ has been used for the SAuNRs and LAuNRs monolayer buildup, the optical density of the SAuNRs monolayer is relatively smaller than that of LAuNRs. This is an indirect effect of the lower ordering of the nanorods in the SAuNRs layer, as a disordered monolayer has a lower packing density than an ordered monolayer. The average AuNRs surface density (and consequently the optical density) is thus higher for the SAUNRS compared to the LAUNRS. ${ }^{50}$

All these data clearly show that the anisotropy in the optical properties directly arises from the alignment of the nanorods/nanowires along one single direction. As the alignment by GIS is more efficient with high aspect ratio nanoobjects, the optical anisotropy is higher for the LAuNRs and AgNWs compared to the SAuNRs.

\section{Electronic transport properties of AgNWs monlayer thin films}

Silver nanowires can also be used for their conductive properties, as transparent conductive thin films of AgNWs can have conductivities and transparency values challenging the ones reached by doped oxide thin films, such as the widely used Indium Tin Oxide (ITO). ${ }^{56}$ In order to probe if the electronic conduction properties of oriented silver nanowires monolayers are anisotropic, we have measured the sheet resistance in the 4-probe configuration in various directions with respect to the nanowire orientation direction. The I(V) curves are characteristic of an Ohmic behaviour (Fig. 4), with a direct proportionality of the measured voltage drop between the two inner pins of the 4-probe head and the current applied between the two outer pins. The sheet resistance can be extracted from the I(V) slopes for each direction, and the values span from $64 \Omega /$ sq. when the current is measured along the orientation direction (black curve in Fig. 4) and $540 \Omega /$ sq. for the perpendicular direction (red curve in Fig. 4), i.e. that the sheet resistance is almost one order of magnitude lower in the nanowire orientation direction compared to the perpendicular one. It must be noted that these low sheet resistance values are obtained at a pretty low nanowire surface density, which 
ensures a high transparency of the samples in the visible range ( 90\% at $550 \mathrm{~nm}$ ).

\section{Conclusions}

We have shown that oriented thin films of 1D-nanoparticles such as nanorods and nanowires can be efficiently oriented using Grazing Incidence Spraying (GIS). The use of gold nanorods and silver nanowires of increasing aspect ratio but with the same surface chemistry allows us to clearly investigate the effect of the nano-object length. We have thus shown that the quality of alignment increases with the length. Accordingly, highest order parameter $\left(\mathbf{S}_{\text {microscopy }}=\mathbf{0 . 8 3}\right)$ was obtained for AgNWs of $A R>100$ followed by LAuNRs of $A R \sim 16$. The high anisotropy in the nanoparticle ordering results in highly anisotropic optical properties: the highest optical anisotropy of $\mathrm{S}_{\text {opt }}=0.76$ is obtained for AgNWs in the near infrared. Finally, we also show that the electronic transport properties of AgNWs oriented thin films are highly anisotropic, with the sheet resistance being almost one order of magnitude lower in the nanowire orientation direction compared to the perpendicular one. Such oriented plasmonic and/or conductive thin films may find applications in a variety of emerging fields, such as optical filters, on-chip waveguides, (bio)chemical sensors, transparent conductive electrodes or metamaterials.

\section{Experimental section}

\section{Materials}

Chemicals such as Hexadecyltrimethylammonium bromide (CTAB H6269, >99.9\%), Hydrogen tetrachloroaurate trihydrate ( $\left.\mathrm{HAuCl}_{4} .3 \mathrm{H}_{2} \mathrm{O},>99.9 \%\right)$, Sodium borohydride $\left(\mathrm{NaBH}_{4},>99 \%\right), \mathrm{L}-$ Ascorbic acid (AA, >99\%), Silver nitrate $\left(\mathrm{AgNO}_{3}, 99 \%\right)$, Hydroquinone (HQ, 99\%) and polymers such as Poly(vinylpyrrolidone) ( $\mathrm{M}_{\mathrm{w}} \sim 10,000$ : PVP10K or $\mathrm{M}_{\mathrm{w}} \sim 40,000$ : $P V P 4 O K$ ), branched Poly(ethyleneimine) solution (50wt\% in $\mathrm{H}_{2} \mathrm{O}$ ) with average $\mathrm{M}_{n} \sim 60,000$ (PEI6OK), were obtained from Sigma Aldrich and were used as received. Nitric acid (68\%) was purchased from Prolabo. Ethylene Glycol (>99\%) was purchased from JT Baker. MilliQ water (18.2 M $2 . \mathrm{cm})$ was used in all our experiments.

\section{Synthesis of Gold Nanorods and Silver Nanowires}

\section{Synthesis of short Gold Nanorods (AR 7, SAuNRs)}

AuNRs of short AR were synthesized using a protocol reported by Zubarev et al. ${ }^{32}$ where the conventional ascorbic acid reducing agent was replaced by hydroquinone. In a first stage, the fast addition of alkaline $\mathrm{NaBH}_{4}$ solution $\left(0.01 \mathrm{M} \mathrm{NaBH}_{4}\right.$ in $0.01 \mathrm{M} \mathrm{NaOH}, 460 \mu \mathrm{L}$ ) to $\mathrm{HAuCl}_{4}-\mathrm{CTAB}$ complex (10 mL mixture containing $0.5 \mathrm{mM}$ gold salt and $0.1 \mathrm{M} \mathrm{CTAB}$ ) leads to the formation of gold seeds indicated by a rapid colour change to brown and this was aged for 30 minutes without any disturbance at $27^{\circ} \mathrm{C}$.

In a second step, $250 \mu \mathrm{L}$ of $\mathrm{HAuCl}_{4}$ (0.1 M stock solution) was added to $49.75 \mathrm{~mL}$ of $0.1 \mathrm{M} \mathrm{CTAB}$ with stirring being continued for 15 minutes at $150 \mathrm{rpm}$. Next, $350 \mu \mathrm{L}$ of $0.1 \mathrm{M} \mathrm{AgNO}_{3}$ and $2500 \mu \mathrm{L}$ of $0.1 \mathrm{M} \mathrm{HQ}$ were added and hand-shaken until the solution became clear. AuNRs formation was initiated by the addition of $800 \mu \mathrm{L}$ of seed solution with vigorous stirring at $1200 \mathrm{rpm}$ for 30 seconds and aged overnight with no stirring at $27^{\circ} \mathrm{C}$. The as-prepared rods were centrifuged twice at 10000 rpm for 30 minutes.

Synthesis of long Gold Nanorods (AR 16, LAuNRs)

High AR AuNRs were synthesized by a typical three-step seed mediated protocol adopted from Huang et al. ${ }^{33}$ In brief, gold seeds were prepared by the one-shot injection of freshly prepared $0.2 \mathrm{M} \mathrm{NaBH}_{4}$ aqueous solution $(450 \mu \mathrm{L})$ into a $10 \mathrm{~mL}$ mixture consisting $0.1 \mathrm{M} \mathrm{CTAB}$ and $0.25 \mathrm{mM} \mathrm{HAuCl}_{4}$ at $27^{\circ} \mathrm{C}$. Seed formation was indicated by a quick colour change from yellow-orange to brown due to the complete reduction of $\mathrm{Au}^{3+}$ by $\mathrm{NaBH}_{4}$. After 5 minutes of vigorous stirring, the CTAB stabilized gold seeds were aged for 2 hours without any disturbance.

Meanwhile, the so-called growth solution was prepared by introducing $25 \mathrm{~mL}$ of $0.25 \mathrm{mM}$ gold solution into $973 \mathrm{~mL}$ of $0.1 \mathrm{M} \mathrm{CTAB}$. This mixture was stirred for 15 minutes at $27^{\circ} \mathrm{C}$ followed by addition of $2 \mathrm{~mL}$ of $1 \mathrm{mM} \mathrm{HNO}_{3}$ in order to modify the $\mathrm{pH}$ value. This $1 \mathrm{~L}$ growth solution was divided as follows: $83.33 \mathrm{~mL}$ are poured into flasks $A \& B$ and the remaining $833.33 \mathrm{~mL}$ into flask $\mathrm{C}$. Then, $463 \mu \mathrm{L}, 463 \mu \mathrm{L}$ and $4.63 \mathrm{~mL}$ of freshly prepared $0.1 \mathrm{M} \mathrm{AA}$ were added to flasks $\mathrm{A}, \mathrm{B}$ and $\mathrm{C}$ respectively. The solutions became clear upon gentle shaking indicating the reduction of $\mathrm{Au}^{3+}$ to $\mathrm{Au}^{+}$. Finally, $7.4 \mathrm{~mL}$ of the seed solution was added into flask $A$ and shaken for 10 seconds. Then, $7.4 \mathrm{~mL}$ of the solution from flask $A$ was quickly added to flask $B$ and shaken for 10 seconds. Then, $74 \mathrm{~mL}$ of solution $B$ was introduced into flask $C$ and shaken for 30 seconds. Appearance of purple colour denotes the formation of gold nanostructures and this became darker with time and finally turned to reddish brown. Flask $\mathrm{C}$ was thermostated for 5 days at $27^{\circ} \mathrm{C}$. Eventually, the upper solution mostly containing spherical gold nanoparticles and by-products were carefully pipetted out. A thin brown layer at the bottom of the flask was collected and dispersed in $50 \mathrm{~mL}$ water. The resulting dispersion was purified by centrifugation at $2000 \mathrm{rpm}$ for 30 minutes twice to remove excess CTAB.

Surface functionalization of both short and long Gold Nanorods

$50 \mathrm{~mL}$ of the CTAB stabilized gold nanorods suspensions (either SAuNRs or LAuNRs) were added dropwise to $500 \mathrm{~mL}$ of a presonicated 10wt\% PVP10K aqueous solution under ultrasonication followed by gentle overnight stirring (150 rpm) at $27^{\circ} \mathrm{C}$. The PVP coated AuNRs were centrifuged at $3000 \mathrm{rpm}$ for 1 hour to remove uncoated PVP. The purple precipitate present on the sidewalls of the tubes were redispersed in ethanol by brief sonication ( 30 seconds). The resulting brown solution was centrifuged in the same conditions to remove excess PVP. The precipitate was finally dispersed in a mixture of $10 \mathrm{~mL}$ water and $2 \mathrm{~mL}$ ethanol. The PVP-coated short and long AuNRs are denoted as SAUNRs-PVP and LAUNRs-PVP respectively.

\section{Synthesis of Silver Nanowires}

Silver nanowires were synthesized by a polyol synthesis ${ }^{57}$ adapted from Wiley et al. ${ }^{34}$ which consists in the reduction of $\mathrm{AgNO}_{3}$ with Ethylene Glycol (EG). In a typical procedure, stock solutions of the different reagents were first freshly prepared in EG: $0.1285 \mathrm{~g}$ of $\mathrm{NaCl}$ were dissolved in $10 \mathrm{~mL} \mathrm{EG}(220 \mathrm{mM})$, 
$0.0405 \mathrm{~g}$ of $\mathrm{Fe}\left(\mathrm{NO}_{3}\right)_{3}$ in $5 \mathrm{mLEG}(33.5 \mathrm{mM}), 0.6720 \mathrm{~g}$ of $\mathrm{AgNO}_{3}$ in $16 \mathrm{~mL}$ of $E G(24.7 \mathrm{mM})$ and $0.6720 \mathrm{~g}$ of PVP in $16 \mathrm{~mL}$ of EG. $78.5 \mathrm{~mL}$ of EG were introduced in a four-neck round bottom flask and stirred at $250 \mathrm{rpm}$. $0.1 \mathrm{~mL}$ of the $\mathrm{NaCl}$ solution and $0.05 \mathrm{~mL}$ of the $\mathrm{Fe}\left(\mathrm{NO}_{3}\right)_{3}$ solution were added into the flask. The solution was homogenized for 10 minutes, and the round bottom flask was placed on a heating mantle with the temperature being measured within the solution, and a condenser was placed on the flask. Two $20 \mathrm{~mL}$ syringes were then filled with $10 \mathrm{~mL}$ of the $\mathrm{AgNO}_{3}$ solution and $10 \mathrm{~mL}$ of the PVP solution respectively. Both solutions were simultaneously injected in the round bottom flask at room temperature under stirring using syringe pumps at a rate of $1.66 \mathrm{~mL} / \mathrm{min}$, i.e. that the total injection time was 6 minutes. After complete addition of $\mathrm{AgNO}_{3}$ and $\mathrm{PVP}$, the temperature was increased up to $192^{\circ} \mathrm{C}$ at a rate of $9^{\circ} \mathrm{C} / \mathrm{min}$. The solution was then cooled to room temperature with a water bath, and was transferred to an Erlenmeyer flask and left undisturbed for two days, in order to allow the longest AgNWs to precipitate. The supernatant was removed and the precipitate was dissolved in $100 \mathrm{~mL}$ of acetone. The AgNWs quickly precipitate, and the supernatant was removed after 5 minutes. This acetone washing procedure without centrifugation was applied twice. Finally, the AgNWs were suspended in $100 \mathrm{~mL}$ of ethanol and were washed three times by centrifugation at $2000 \mathrm{rpm}$ for 30 minutes. Finally AgNWs were kept in ethanol for further use.

\section{Oriented deposition of anisotropic nanoparticles}

\section{Substrate preparation}

Oriented 1D-nanoparticle thin films were deposited on glass substrates as it allows polarized UV-Vis-NIR spectroscopy measurements. All glass substrates were first cleaned by initial blowing with $\mathrm{N}_{2}$ and subsequent sonication in water for 10 minutes followed by copious ethanol rinsing, drying under a $\mathrm{N}_{2}$ stream and plasma activation for 3 minutes. The glass substrates were then coated with a PEI6OK $\left(2.5 \mathrm{mg} / \mathrm{mL}\right.$ in $\left.\mathrm{H}_{2} \mathrm{O}\right)$ layer by spraying for 10 seconds and subsequently spray rinsed with water for the same time. Manual air-pump spray cans (Roth) were used for the PEI deposition and for rinsing.

\section{Grazing Incidence Spraying}

Oriented monolayers of 1D- metal nanoparticles were deposited on the PEI-coated glass slides by Grazing Incidence Spraying (GIS) 29, 30 using a semi-automated home-made setup equipped with a two-fluid spray nozzle (Spraying Systems, internal diameter: $0.3 \mathrm{~mm}$, for AuNRs and Aztek A480 airbrush, internal diameter: $1.03 \mathrm{~mm}$, for AgNWs). The angle between the central axis of the cone of the spray jet and the receiving substrate was fixed at $\alpha=15-20^{\circ}$, and the distance between the nozzle and the substrate was held at a constant distance of $1 \mathrm{~cm}$. The liquid flow rate was fixed to $1 \mathrm{~mL} / \mathrm{min}$ and the air flow rate to $40 \mathrm{~L} / \mathrm{min}$ for AuNRs and $30 \mathrm{~L} / \mathrm{min}$ for $\mathrm{AgNWs}$. which produces a cloud of droplets with a diameter in the range of $5-20 \mu \mathrm{m}$ as determined by Phase Doppler Interferometry (PDI, data not shown here). Following deposition, the thin film was rinsed with Milli-Q water in order to remove any weakly adsorbed nanoparticles and dried using a $\mathrm{N}_{2}$ flow.

\section{Characterization}

Electron Microscopy
TEM was performed by depositing $10 \mu \mathrm{L}$ of the colloidal suspension on a carbon coated copper grid which is left to to dry in air for a few hours. The nanoparticles were imaged with a Tecnai G2 FEl microscope operated at 120kV. AgNWs, as well as the oriented monolayer thin films were imaged by SEM using a JEOL 6700F microscope operating at $1 \mathrm{kV}$ and $10 \mu \mathrm{A}$. The orientation analysis was done on SEM pictures using OrientationJ, ${ }^{54}$ a plugin developed for ImageJ, 55 which is based on the computation of the structure tensor in the local neighbourhood of each pixel. The original SEM picture is first binarized, and the OrientationJ plugin is applied with a Gaussian gradient on a window of 3 pixels. As a result, an orientation angle is given for each pixel, and a color-code which represents each angle by a color is used to build the images given in Fig. 2C. Finally, the angle distribution with respect to the main orientation direction can be extracted (Fig. 2D). The angle distribution is non-weighted (i.e. 1 pixel $=1$ count), but the pixels for which the values of the coherency or the energy are lower than $10 \%$ have been ignored. Indeed, these pixels are often artifacts, for instance noisy pixels or located at edges and corners.

\section{UV-Vis-NIR spectroscopy}

UV-Visible-Near Infrared spectroscopy was carried out with a Cary 5000 spectrometer (Agilent) in dual beam configuration. The extinction for the thin film samples was measured through a circular aperture (diameter $=5 \mathrm{~mm}$ ) and a blank substrate was used as a reference. A Glan-Taylor polarizer has been used for polarized UV-Vis-NIR spectroscopy.

\section{Sheet resistance measurements}

The oriented $\mathrm{AgNWs}$ were annealed at $175^{\circ} \mathrm{C}$ for 1 minute and 45 seconds in order to reduce the NW/NW contact resistance. ${ }^{58}$ The sheet resistance was measured on monolayer thin films using a 4-probe head (Signatone 302 Resistivity Stand) connected to a Keithley 2400 sourcemeter. I(V) sweeps were obtained by applying current between the two outer pins ($50 \mu \mathrm{A}<\mathrm{I}<50 \mu \mathrm{A})$ and measuring the voltage drop between the inner pins. The sheet resistance can be calculated from the I(V) slope using:

$$
R_{S}=4.5324 \frac{d V}{d I} \quad \text { Eq. } 4
$$

\section{Acknowledgements}

The authors thank Marc Schmutz and Christian Blanck (ICS, France) for TEM measurements, Cedric Leuvrey (IPCMS, France) for SEM measurements. The sheet resistance measurements were done in the STnano cleanroom facility in Strasbourg, France. S.S. thanks the IDEX program of the Strasbourg University for funding. V.L. thanks the Labex CSC "Chemistry of Complex Systems" for financial support. M.P. thanks the "Chaire d'Excellence" program of CNRS and the Université de Strasbourg for partial funding. G.D. thanks the Institut Universitaire de France for financial support.

\section{Notes and references}

1. P. R. Sajanlal, T. S. Sreeprasad, A. K. Samal and T. Pradeep, Nano Rev., 2011, 2, 5883. 
2. K. Thorkelsson, P. Bai and T. Xu, Nano Today, 2015, 10, 48-66.

3. G. Decher, Science, 1997, 277, 1232-1237.

4. J. Schmitt, G. Decher, W. J. Dressick, S. L. Brandow, R. E. Geer, R. Shashidhar and J. M. Calvert, Adv. Mater., 1997, 9, 61-65.

5. S. Srivastava and N. A. Kotov, Acc. Chem. Res., 2008, 41, 18311841.

6. G. Schneider, G. Decher, N. Nerambourg, R. Praho, M. H. V. Werts and M. Blanchard-Desce, Nano Lett., 2006, 6, 530-536.

7. B. P. Pichon, P. Louet, O. Felix, M. Drillon, S. Begin-Colin and G. Decher, Chem. Mater., 2011, 23, 3668-3675.

8. O. L. Muskens, G. Bachelier, N. D. Fatti, F. Vallée, A. Brioude, X. Jiang and M.-P. Pileni, J. Phys. Chem. C, 2008, 112, 8917-8921. 9. Z. Liu, J. Xu, D. Chen and G. Shen, Chem. Soc. Rev., 2015, 44, 161-192.

10. Y. Xia, P. Yang, Y. Sun, Y. Wu, B. Mayers, B. Gates, Y. Yin, F. Kim and H. Yan, Adv. Mater., 2003, 15, 353-389.

11. S. Lal, J. H. Hafner, N. J. Halas, S. Link and P. Nordlander, Acc. Chem. Res., 2012, 45, 1887-1895.

12. L. Guerrini and D. Graham, Chem. Soc. Rev., 2012, 41, 70857107.

13. K. A. Willets, Chem. Soc. Rev., 2014, 43, 3854-3864.

14. A. Lee, G. F. S. Andrade, A. Ahmed, M. L. Souza, N. Coombs, E. Tumarkin, K. Liu, R. Gordon, A. G. Brolo and E. Kumacheva, J. Am. Chem. Soc., 2011, 133, 7563-7570.

15. H. Wei and H. Xu, Nanoscale, 2013, 5, 10794-10805.

16. A. Campion and P. Kambhampati, Chem. Soc. Rev., 1998, 27 241-250.

17. H. Chen, L. Shao, Q. Li and J. Wang, Chem. Soc. Rev., 2013, 42, 2679-2724.

18. L. Duempelmann, A. Luu-Dinh, B. Gallinet and L. Novotny, ACS Photonics, 2015, DOI: 10.1021/acsphotonics.5b00604.

19. A. Klinkova, R. M. Choueiri and E. Kumacheva, Chem. Soc. Rev., 2014, 43, 3976-3991.

20. L. Mai, Y. Gu, C. Han, B. Hu, W. Chen, P. Zhang, L. Xu, W. Guo and Y. Dai, Nano Letters, 2009, 9, 826-830.

21. S. Raychaudhuri, S. A. Dayeh, D. Wang and E. T. Yu, Nano Lett. 2009, 9, 2260-2266.

22. C. M. Hangarter and N. V. Myung, Chem. Mater., 2005, 17 $1320-1324$

23. Y. Huang, X. Duan, Q. Wei and C. M. Lieber, Science, 2001, 291 630-633.

24. J. Pérez-Juste, B. Rodríguez-González, P. Mulvaney and L. M. Liz-Marzán, Adv. Funct. Mater., 2005, 15, 1065-1071.

25. G. Yu, X. Li, C. M. Lieber and A. Cao, Journal of Materials Chemistry, 2008, 18, 728-734.

26. G. Yu, A. Cao and C. M. Lieber, Nat Nano, 2007, 2, 372-377.

27. O. Assad, A. M. Leshansky, B. Wang, T. Stelzner, S. Christiansen and H. Haick, ACS Nano, 2012, 6, 4702-4712.

28. G. Decher, R. Blell, H. Hu, M. Pauly, O. Felix, D. Martel, X. Lin, S. Sekar, S. Diabang, J. Baer US Pat., WO2015173432 A1, 2015.

29. H. Hu, M. Pauly, O. Felix and G. Decher, submitted, 2016.

30. R. Blell, X. Lin, T. Lindström, M. Ankerfors, M. Pauly, O. Félix and G. Decher, submitted, 2016.

31. E. Seyrek and G. Decher, in Polymer Science: A Comprehensive Reference, ed. K. M. Möller, Elsevier, Amsterdam, 2012, vol. 7, pp. 159-185.

32. L. Vigderman and E. R. Zubarev, Chem. Mater., 2013, 25, 14501457.

33. Y.-N. Wang, W.-T. Wei, C.-W. Yang and M. H. Huang, Langmuir, 2013, 29, 10491-10497.

34. S. M. Bergin, Y.-H. Chen, A. R. Rathmell, P. Charbonneau, Z.-Y. Li and B. J. Wiley, Nanoscale, 2012, 4, 1996-2004.

35. B. D. Busbee, S. O. Obare and C. J. Murphy, Advanced Materials, 2003, 15, 414-416.

36. B. Nikoobakht, Z. L. Wang and M. A. El-Sayed, The Journal of Physical Chemistry B, 2000, 104, 8635-8640.

37. X. M. Sun and Y. D. Li, Adv. Mater., 2005, 17, 2626-2630.

38. M. S. Goh, Y. H. Lee, S. Pedireddy, I. Y. Phang, W. W. Tjiu, J. M.

R. Tan and X. Y. Ling, Langmuir, 2012, 28, 14441-14449.
39. M. Chen, I. Y. Phang, M. R. Lee, J. K. W. Yang and X. Y. Ling, Langmuir, 2013, 29, 7061-7069.

40. J. P. Kottmann, O. J. F. Martin, D. R. Smith and S. Schultz, Phys. Rev. B, 2001, 64, 235402.

41. X. Ye, L. Jin, H. Caglayan, J. Chen, G. Xing, C. Zheng, V. DoanNguyen, Y. Kang, N. Engheta, C. R. Kagan and C. B. Murray, ACS Nano, 2012, 6, 2804-2817.

42. B. Nikoobakht and M. A. El-Sayed, Chem. Mater., 2003, 15 , 1957-1962.

43. L. Vigderman, B. P. Khanal and E. R. Zubarev, Adv. Mater., 2012, 24, 4811-4841.

44. S. Link, M. B. Mohamed and M. A. El-Sayed, The Journal of Physical Chemistry B, 1999, 103, 3073-3077.

45. K. Park, H. Koerner and R. A. Vaia, Nano Letters, 2010, 10, 1433-1439.

46. B. P. Khanal and E. R. Zubarev, Journal of the American Chemical Society, 2008, 130, 12634-12635.

47. N. R. Jana, Chemical Communications, 2003, DOI: 10.1039/B303103A, 1950-1951.

48. W. A. Saidi, H. Feng and K. A. Fichthorn, J. Phys. Chem. C, 2012, 117, 1163-1171.

49. S. Gómez-Graña, F. Hubert, F. Testard, A. Guerrero-Martínez, I. Grillo, L. M. Liz-Marzán and O. Spalla, Langmuir, 2012, 28, 14531459.

50. S. Vial, I. Pastoriza-Santos, J. Pérez-Juste and L. M. Liz-Marzán, Langmuir, 2007, 23, 4606-4611.

51. I. Pastoriza-Santos, D. Gomez, J. Perez-Juste, L. M. Liz-Marzan and P. Mulvaney, Physical Chemistry Chemical Physics, 2004, 6, 5056-5060.

52. T. Hendel, M. Wuithschick, F. Kettemann, A. Birnbaum, K. Rademann and J. Polte, Analytical Chemistry, 2014, 86, 1111511124.

53. L. Scarabelli, A. Sánchez-Iglesias, J. Pérez-Juste and L. M. LizMarzán, The Journal of Physical Chemistry Letters, 2015, 6, 42704279.

54. R. Rezakhaniha, A. Agianniotis, J. T. C. Schrauwen, A. Griffa, D. Sage, C. V. C. Bouten, F. N. Vosse, M. Unser and N. Stergiopulos, Biomech. Model. Mechanobiol., 2012, 11, 461-473.

55. C. A. Schneider, W. S. Rasband and K. W. Eliceiri, Nat. Meth., 2012, 9, 671-675.

56. S. Ye, A. R. Rathmell, Z. Chen, I. E. Stewart and B. J. Wiley, Adv. Mater., 2014, 26, 6670-6687.

57. Y. Sun, Y. Yin, B. T. Mayers, T. Herricks and Y. Xia, Chem. Mater., 2002, 14, 4736-4745.

58. D. P. Langley, M. Lagrange, G. Giusti, C. Jimenez, Y. Brechet, N. D. Nguyen and D. Bellet, Nanoscale, 2014, 6, 13535-13543. 